\section{LA-UR-03-3154}

Approved for public release; distribution is unlimited.

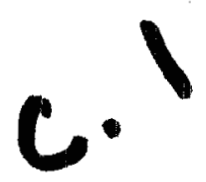

\section{Title: \\ A NUMERICAL STUDY OF BUNCHED BEAM TRANSVERSE e-p INSTABLILITY BASED ON THE CENTROID MODEL}

Author(s):

Tai-Sen F. Wang, LANSCE-1

2003 Particle Accelerator Conference (PAC2003)

Portland, Oregon

May $12-16,2003$
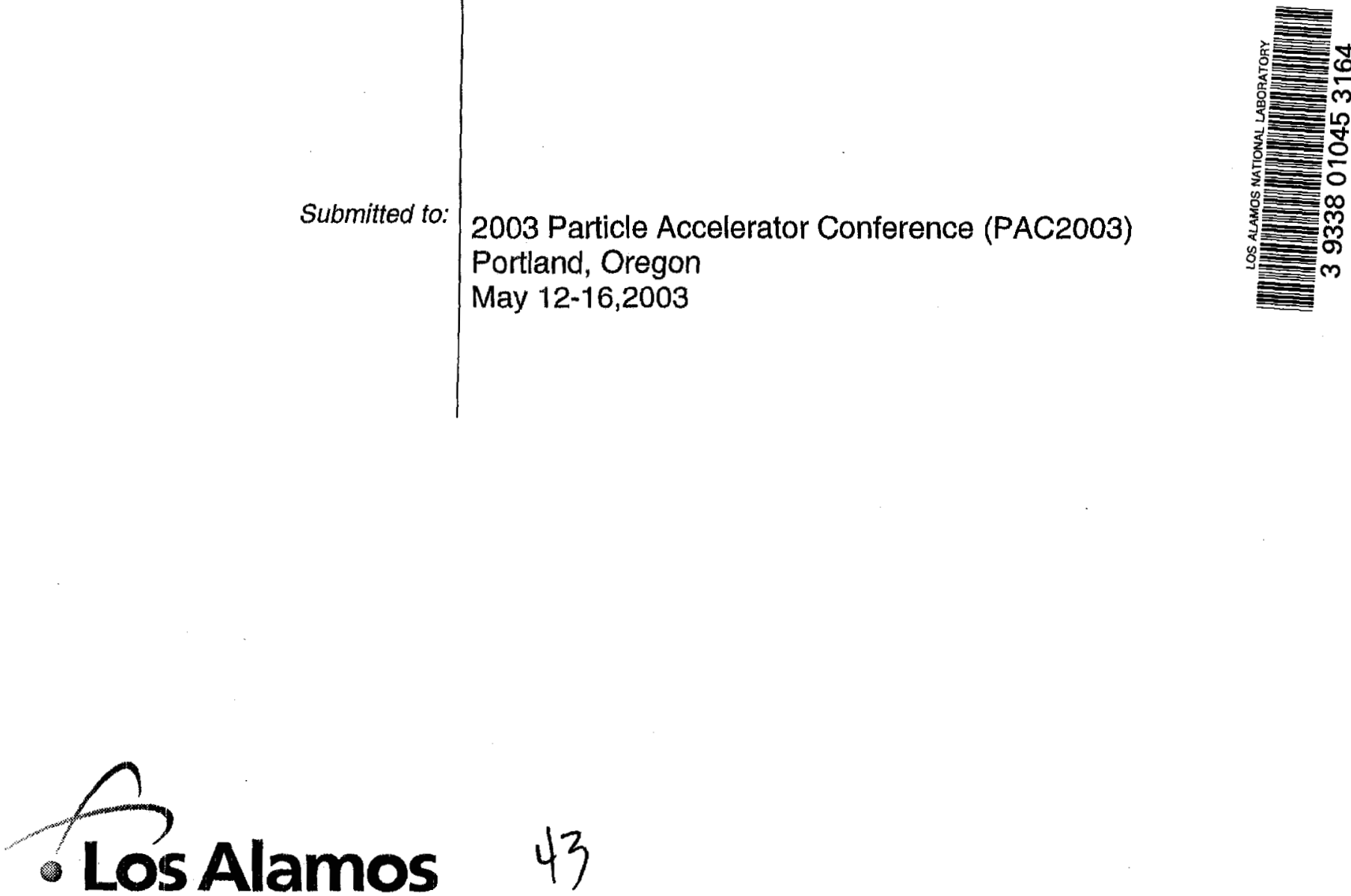

43

NATIONAL LABOAATORY

Los Alamos National Laboratory, an affirmative action/equal opportunity employer, is operated by the Un $\equiv$ ity of California for the U.S. Department of Energy under contract W-7405-ENG-36. By acceptance of this article, the publisher reco $D$ s that the U.S. Government retains a nonexclusive, royalty-free license to publish or reproduce the published form of this contribution, or to allow others to do so, for U.S. Government purposes. Los Alamos National Laboratory requests that the publisher identify this article as work performed under the auspices of the U.S. Department of Energy. Los Alamos National Laboratory strongly supports academic freedom and a researcher's right to publish; as an institution, however, the Laboratory does not endorse the viewpoint of a publication or guarantee its technical correctness. 


\title{
A NUMERICAL STUDY OF BUNCHED BEAM TRANSVERSE $e-p$ INSTABILITY BASED ON THE CENTROID MODEL *
}

\author{
Tai-Sen F. Wang ${ }^{\dagger}$, LANL, Los Alamos, NM 87545, USA
}

\section{Abstract}

In a recent theoretical study of the transverse electronproton $(e-p)$ instability, an asymptotic solution has been found for the equations describing the centroid motion of the traversing proton bunch and the stationary background electrons.[1] It was shown that the combination of finite proton bunch length, non-uniform proton line density, and the single-pass e-p interaction cause the instability to evolve intricately in space and time even in the linear regime. This paper reports a numerical study of the $e-p$ instability based on the same centroid equations. The purpose of the work is to compare the numerical solution with the analytic solution and to use the numerical approach to investigate the early development of the instability not covered by the asymptotic solution. In particular, the instability threshold and the initial growth of the instability are studied for various proton-beam conditions, fraction of charge neutralization, and initial perturbations.

\section{INTRODUCTION}

In recent years, there has been a growing interest in studying the transverse electron-proton $(e-p)$ two-stream instability in intense proton beams. One of the focuses is on the $e-p$ instability observed in the long proton bunch like the one in the Proton Storage Ring (PSR) at Los Alamos National Laboratory [2]. Although the basic mechanism of the e-p instability has been well known, the theory for a bunched beam e-p instability is still under developing. In a recent theoretical study of transverse $e-p$ instability, an asymptotic solution has been found for the equations describing the centroid motion of the traversing proton bunch and the stationary background electrons.[1] It was shown that the combination of finite proton bunch length, nonuniform line densities, and the single-pass $e-p$ interaction cause the instability to evolve intricately in space and time even in the linear regime. This paper reports a numerical study of the $e-p$ instability based on the same centroid equations. The purpose of the work is to compare the numerical solution with the analytic solution and to use the numerical approach to investigate the early development of the instability not covered by the asymptotic solution. In particular, the instability threshold and the initial growth of the instability are studied for various proton-beam conditions, fraction of charge neutralization, and initial perturbations.

\footnotetext{
* Research supported by Los Alamos National Laboratory under the auspices of the US Department of Energy.

†TWANG@LANL.GOV
}

\section{CENTROID MODEL}

We consider a bunched proton beam of full length $L$ and circular cross section of radius $a$, propagating with a constant velocity $v$ through a stationary electron background of infinite extent in the direction of beam propagation. We assume that in the equilibrium state, the electrons are trapped in the proton beam, and the particles are distributed uniformly in the transverse direction so the trapped electrons experience a linear transverse focusing force. The protons are confined in the transverse direction by a linear external focusing force. A Cartesian coordinate system is chosen such that the $z$ axis is pointing opposite to the direction of proton propagation, and the origin coincides with the center of the beam cross section. The line densities of the protons and electrons, $\lambda_{p}$ and $\lambda_{e}$, generally depend on $z$. The synchrotron motion of the protons and the axial motion of the electrons in the laboratory frame are neglected for simplicity. We also neglect the impedance due to the beam environment, and consider the transverse motion in only one direction, say the $y$ direction. The stability study is based on a model in which each electron interacts with the proton beam only once, i.e., a "one-pass" interaction between the electrons and protons.

The centroid of the proton beam $Y_{p}(z, t)$ and the centroid of electrons $Y_{e}(z, t)$ are defined by

$$
Y_{q}(z, t)=\int_{-\infty}^{\infty} y_{q}\left(z, t, \omega_{q}\right) F_{q}\left(\omega_{q}\right) d\left(\omega_{q} / \Delta_{q}\right)
$$

where the subscripts $q$ stands for $p$ (protons) or $e$ (electrons), $y_{q}\left(z, t, \omega_{q}\right)$ is the particle displacement at the position $z$ and time $t, \omega_{q}$ is the oscillation frequency, $F_{q}\left(\omega_{q}\right)$ is the frequency distribution function, and $\Delta_{q}$ characterizes the frequency spread of $\omega_{q}$. We consider a Lorentzian distribution function $F_{q}\left(\omega_{q}\right)=\left(\Delta_{q}^{2} / \pi\right)\left[\Delta_{q}^{2}+\left(\omega_{q}-\omega_{q o}\right)^{2}\right]^{-1}$, where $\omega_{q o}$ is the mean value of $\omega_{q}$. Averaging over the equations of single particle motion yields

$$
D^{2} Y_{p}+2 \Delta_{p} D Y_{p}+\left(\omega_{\beta}^{2}+\Delta_{p}^{2}\right) Y_{p}=\omega_{\beta}^{2} \xi(z) Y_{e},
$$

and

$$
\ddot{Y}_{e}+2 \Delta_{e} \dot{Y}_{e}+\left[\Omega^{2}(z)+\Delta_{e}^{2}\right] Y_{e}=\Omega^{2}(z) Y_{p},
$$

where $D=\partial / \partial t-v(\partial / \partial z)$, and $\omega_{\beta}$ is the undepressed betatron frequency, $\xi(z)=2 r_{p} c^{2} \lambda_{e}(z) /\left(a^{2} \omega_{\beta}^{2} \gamma\right), \Omega(z)=$ $(c / a) \sqrt{2 r_{e} \lambda_{p}(z)}$, is the electron bounce frequency, $c$ is the speed of light, $\gamma=\left(1-v^{2} / c^{2}\right)^{-1 / 2} ; r_{p}$ and $r_{e}$ are the classical radii of a proton and an electron, respectively. In deriving Eqs. (2) and (3), we have also assumed that the incoherent betatron frequency shift due to the self-fields of the proton beam is negligible, and that the maximum value of $\lambda_{e}$ is much smaller than that of $\lambda_{p}$, so that $\omega_{p o}=\omega_{\beta}$. The 
perturbing forces are assumed to be $m_{e} \Omega^{2}(z) Y_{p}$ for electrons and $m_{p} \omega_{\beta}^{2} \xi(z) Y_{e}$ for protons, where $m_{q}$ is the relativistic mass of a proton or an electron. Note that Eqs. (2) and (3) depend on the choice of the frequency distribution function.

An approximate asymptotic solution for Eqs. (2) and (3) in the beam frame is found to be

$$
\begin{gathered}
Y_{p}\left(z^{\prime}, t\right) \approx C_{p} M_{p}\left(z^{\prime}\right) \mathrm{e}^{-\Delta_{p} t}\left\{\left[\frac{I_{1}(u)}{u}-\frac{\mathcal{J}^{2} I_{2}(u)}{8 u^{2}}\right]\right. \\
\left.\times \cos T_{p}-\left[\frac{J_{1}(u)}{u}-\frac{\mathcal{J}^{2} J_{2}(u)}{8 u^{2}}\right] \cos S_{p}\right\},
\end{gathered}
$$

where $z^{\prime}$ is the distance from the head of the proton bunch, $J_{n}(x)$ and $I_{n}(x)$ are Bessel functions, $u=\sqrt{2 \theta \mathcal{J}}, \theta=$ $\theta\left(z^{\prime}, t\right)=\omega_{\beta}\left(t-z^{\prime} / v\right), M_{p}\left(z^{\prime}\right)=\mathcal{J} \xi\left(z^{\prime}\right) R\left(z^{\prime}\right) \exp \left[\left(\Delta_{p}-\right.\right.$ $\left.\left.\Delta_{e}\right) z^{\prime} / v\right], T_{p}=P-\theta, S_{p}=P+\theta, P=\sigma_{p}+\Theta\left(z^{\prime}\right)-\mathcal{J} / 4$, $C_{p}$ and $\sigma_{p}$ are constants, $R\left(z^{\prime}\right)$ and $\Theta\left(z^{\prime}\right)$ are determined by $\Phi(x)=R(x) \mathrm{e}^{i \Theta(x)}$ and $\Psi(x)=R(x) \mathrm{e}^{-i \Theta(x)}$,

$$
\mathcal{J}=\mathcal{J}\left(z^{\prime}\right)=i \int_{0}^{z^{\prime} / v} \frac{\Omega^{2}(x) \xi(v x)}{W(x)}[R(x)]^{2} d x,
$$

$i=\sqrt{-1}, \Phi(x)$ and $\Psi(x)$ are the linearly independent solutions of the equation $d^{2} Y / d x^{2}+\Omega^{2}(x) Y=0$, and $W(x)$ is the Wronskian of $\Phi(x)$ and $\Psi(x)$. In deriving Eq. (4), we have assumed that $Y_{e}=d Y_{e} / d t=0$ for $z^{\prime} \leq 0$. The solution for $Y_{e}$ is very similar. The approximate growth rate or damping rate $\Gamma_{p}\left(z^{\prime}, t\right)$, and the instability threshold of the proton motion $\left(\Delta_{p}\right)_{t}$, can be estimated from $\mathrm{Eq}$. (4) as

$$
\Gamma_{p}\left(z^{\prime}, t\right) \approx-\Delta_{p}+\frac{\omega_{\beta} \mathcal{J}\left[8 u I_{2}(u)-\mathcal{J}^{2} I_{3}(u)\right]}{u\left[8 u I_{1}(u)-\mathcal{J}^{2} I_{2}(u)\right]}
$$

and

$$
\left(\Delta_{p}\right)_{t} \approx \operatorname{Max}\left[\frac{\omega_{\beta} \mathcal{J}}{4}\left(\frac{1-\mathcal{J}^{2} / 48}{1-\mathcal{J}^{2} / 32}\right)\right],
$$

where $\left[Y_{p}\left(z^{\prime}, t\right)\right]_{a}$ denotes the amplitude of $Y_{p}\left(z^{\prime}, t\right)$, and $\operatorname{Max}\left[f\left(z^{\prime}\right)\right]$ indicates the maximum of $f\left(z^{\prime}\right)$ for $0 \leq z^{\prime} \leq$ $L$. For $u \gg 1$, we have $\Gamma_{g}\left(z^{\prime}, t\right) \approx \omega_{\beta} \sqrt{\mathcal{J} /(2 \theta)}-\Delta_{p}$. Typically, $\mathcal{J}<1$ for a small fractional charge neutralization, $\Gamma_{p}\left(z^{\prime}, t\right)$ is a monotonically decreasing functions of time, and the largest growth rate and the lowest threshold are at the tail of the proton bunch. Equation (7) is valid for small $\mathcal{J}$.

\section{NUMERICAL STUDY}

Equations (2) and (3) were solved numerically for stability study. Previous numerical studies as well as simulations carried out using equations similar to Eqs. (2) and (3), including variantions that cover multi-electrons and electron production, have yield reasonable agreement with experimental data. $[3,4]$

Comparison between numerical results at large time and the asymptotic solution given in Eq. (4) shows good qualitative agreement. The growth rate depends linearly on $\Delta_{p}$ is a consequence of adopting the Lorenzian frequency spreads. The perturbation wavelength along the proton bunch is roughly proportional to the square root of the proton line density. The instability damps in the long time as indicated in the asymptotic solution. At largre $t$, the growth or the damping rate depend weakly on $\Delta_{e}$.

Our main interest is to use Eqs. (2) and (3) to study the initial evolution of perturbations. The following is a summary of our observation on many numerical results:

(i) As expected, the linear dependence of the growth rate on $\Delta_{p}$ is not affected by initial conditions.

(ii) The initial growth or damping rate does depend on $\Delta_{e}$. The dependence deminishes as time increases. This result is not covered by the asymptotic solution in Eq. (4).

(iii) Since the growth rate given in Eq. (6) is a local quantity, i.e. depending on space and time, and the model we are considering has the one-pass interaction between the electrons and the protons, initial perturbations with quarter wavelength comparable to the bunch length have strong influence on the local growth rate initially. Further, the growth rate at any location is not affected by the perturbations behind that location. Typically, perturbations having high frequency structures (wavelength substantially smaller than the bunch length) with constant envelope are initially damped at a rate near $\Delta_{p}$. For perturbations having tilted envelope, both the magnitude and the slope of the initial damping rate are shifted from $\Delta_{p}$ by certain amounts with a sign opposite to that of envelope's slope.

(iv) The growth rate (or the damping rate) may oscillate before it evolves into the asymptotic regime. The systen stability can not be judged on the momentary sign of the growth rate.

(v) The time evolution of the growth rate depends on the density profile and other parameters in a complicated way. So far, we still do not have any general conclusion on this aspect. It is observed that the transient duration for the growth rate to evolve into the asymptotic regime is independent of $\Delta_{p}$. Variations of other parameter value that make the system less stable tend to shorten the transient period.

(vi) The initial growth rate estimated in Eq. (6) is usually much higher than the simulation results. Estimate of threshold made by using Eq. (7) is too conservative in general as discussed in Ref. 3.

To give an example of many trials in our study, we concentrate on a few specific density profiles and initial conditions with frequency spread and intensity chosen near the instability threshold. Thus, we considered a constant electron line density and four proton line densities: constant, elliptical, parabolic, and quartic (parabolic squared) line densities. Four types of initial conditions of proton centroid were studied: wavelength proportional to $\sqrt{\lambda_{p}}$, noise, $100 \mathrm{MHz}\left(<f_{e m}\right)$, and $250 \mathrm{MHz}\left(>f_{e m}\right)$, where $f_{e m}$ is the peak electron bounce frequency in the proton bunch. All these four initial perturbation have a same constant envelope. The electron centroid was assumed to be unperturbed when entering the proton bunch. The following PSR 
parameter values were used for computation: $\gamma=1.85$, $a=1.5 \mathrm{~cm}$, the circumference $C=90 \mathrm{~m}, 2.74 \times 10^{13}$ protons per bunch, $\omega_{\beta}=40 \mathrm{MHz}$, and $L / v=200 \mathrm{~ns}$ (for a short bunch). The proton and electron oscillation frequency spreads are chosen as $\Delta_{p} / \omega_{\beta}=0.125 \%$ and $\Delta_{e} / \omega_{\beta}=1.25$, respectively. We also assume $2 \%$ of neutralization at the center of the proton bunch.

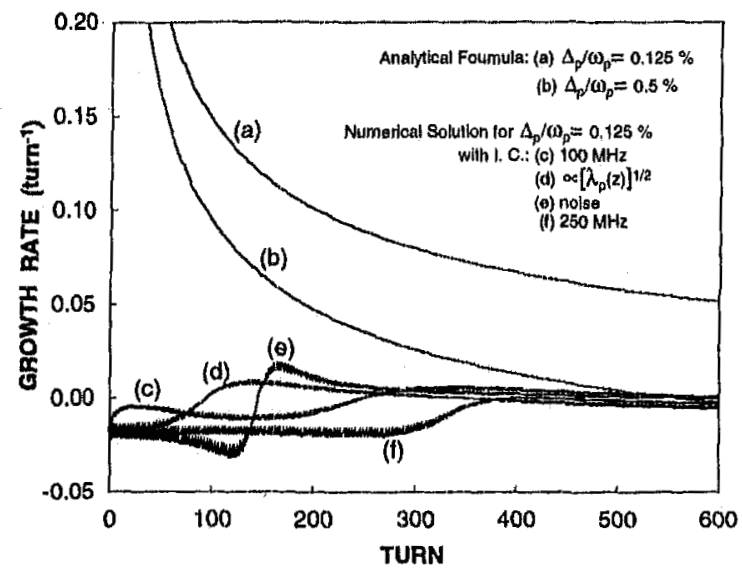

Figure 1: The growth rate at the tail of the proton bunch as a function of turn in PSR for different initial conditions and the growth rate computed using the asymptotic formula (6). Parabolic proton line density is considered here.

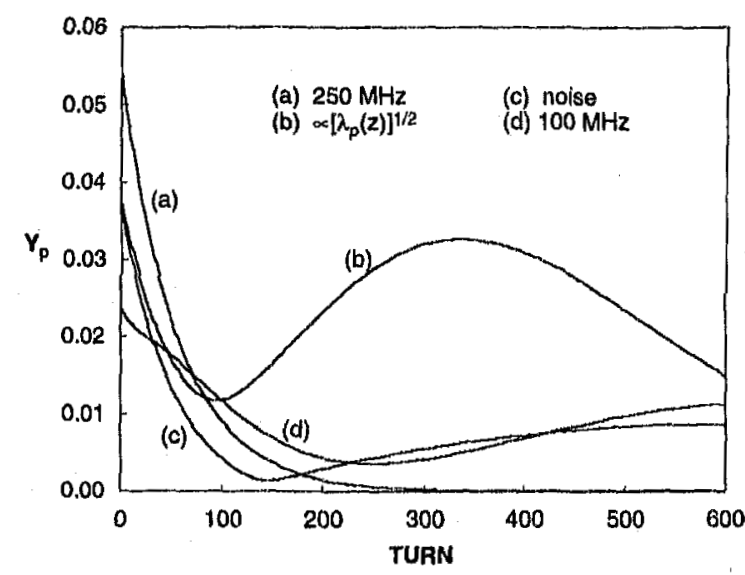

Figure 2: The amplitude of oscillation for the proton centroid is shown as a function of turn in PSR for the cases considered in Fig. 1. The ordinate has an arbitrary unit.

Shown in Fig. 1 is the growth rate at the tail of the proton bunch as a function of turn in PSR for different initial conditions and parabolic proton line density. The growth rate computed using the asymptotic formula is also shown for comparison. The corresponding amplitude of proton centroid oscillation as a function of turn is shown in Fig. 2. It is seen that the initial perturbation with wavelength proportional to $\sqrt{\lambda_{p}}$ is the least stable one among the four initial conditions considered. Figure 3 shows the growth rate at the tail of the proton bunch as a function of turn for differ- ent proton line densities and a same noise initial condition.

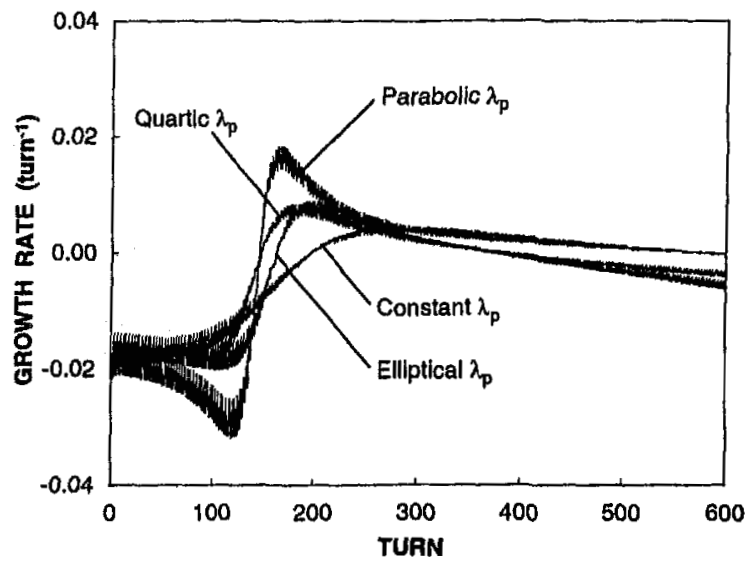

Figure 3: The growth rate at the tail of the proton bunch as a function of turn in PSR for different proton line densities and a same noise initial condition.

\section{CONCLUSIONS}

We have carried out a numerical study of the initial evolution of the transverse $e-p$ instability based on the equations describing the motion of the proton centroid and the electron centroid. The model considered is based on the Lorentzian distribution of particles' oscillation frequencies and the one-pass interaction between the stationary electrons and traveling proton bunch. We have studied various proton line densities and initial perturbations in the proton centroid. We have also compared the numerical solutions with the asymptotic solution derived analytically and found good qualitative agreements between these two solutions when the time is large. We found that the initial evolution of the instability depends on the proton line density and the initial conditions. The growth rate and the threshold estimated from the asymptotic solution over estimate and too conservative for general cases. We also found that the combination of finite proton bunch length, non-uniform proton line density, and the one-pass e-p interaction cause the instability to evolve intricately in space and time even in the linear regime.

\section{REFERENCES}

[1] T. Wang, P. J. Channell, R. J. Macek, and R. C. Davidson, Phys. Rev. ST Accel. Beams, 6, 014204 (2003).

[2] Articles in Two Stream Special Collection, Phys. Rev. STAB, Ed. R. C. Davidson and R. J. Macek, and the references cited therein.

[3] T. Wang, AIP Conf. Proc. 496, p. 305 (1999).

[4] T. Wang, Proc. of 1995 Particle Accelerator Conf., p. 3143. 